\title{
USING SUBNORMALITY TO SHOW THE SIMPLE CONNECTIVITY AT INFINITY OF A FINITELY PRESENTED GROUP
}

\author{
JOSEPH S. PROFIO
}

\begin{abstract}
A CW-complex $X$ is simply connected at infinity if for each compact $C$ in $X$ there exists a compact $D$ in $X$ such that loops in $X-D$ are homotopically trivial in $X-C$. Let $G$ be a finitely presented group and $X$ a finite CW-complex with fundamental group $G . G$ is said to be simply connected at infinity if the universal cover of $X$ is simply connected at infinity. B. Jackson and C. M. Houghton have independently shown that if $G$ and a normal subgroup $H$ are infinite finitely presented groups with $G / H$ infinite and either $H$ or $G / H$ 1-ended, then $G$ is simply connected at infinity. In the case where $H$ is 1-ended, we exhibit a class of groups showing that the "finitely presented" hypothesis on $H$ cannot be reduced to "finitely generated." We address the question: if $N$ is normal in $H$ and $H$ is normal in $G$ and these are infinite groups with $N$ and $G$ finitely presented and either $N$ or $G / H$ is 1-ended, is $G$ simply connected at infinity? In the case that $N$ is 1-ended, the answer is shown to be yes. In the case that $G / H$ is 1 -ended, we exhibit a class of such groups that are not simply connected at infinity.
\end{abstract}

\section{INTRODUCTION}

The purpose of this paper is to extend a result of Jackson [7] and Houghton [6] on group extensions that are simply connected at $\infty$. In [7 and 6] we find

Theorem. Let $H \triangleleft G$ be infinite finitely presented groups with $G / H$ infinite. If $H$ or $G / H$ is 1-ended, then $G$ is simply connected at $\infty$.

If the finitely presented hypothesis on $H$ is reduced to finitely generated, the hypotheses are no longer sufficient to ensure that $G$ will be simply connected at $\infty$. $\S \mathrm{V}$ will exhibit a class of such groups. In particular, let $F$ denote the free group on two generators. $\S \mathrm{V}$ will present a finitely generated, 1-ended normal subgroup $H$ of $F \times F$ such that $(F \times F) / H$ is isomorphic to the group $\mathbf{Z}$ of integers. Furthermore, $F \times F$ is not simply connected at $\infty$.

The question arises as to what extent the hypotheses of Jackson and Hough ton's theorem may be altered while still ensuring that $G$ is simply connected at $\infty$. In [10], Mihalik shows the following:

Received by the editors January 20, 1988 and, in revised form, September 26, 1988.

1980 Mathematics Subject Classification (1985 Revision). Primary 20E99, 20F32, 55P99, 57M10; Secondary 20F05, 20G10, 57M20.

Key words and phrases. Universal cover, CW-complex, group representations, and homotopy. 
Theorem. Let $H \triangleleft G$ be infinite groups with $G$ finitely presented and $G / H$ ended. If $H$ is abelian with an element of infinite order, then $G$ is simply connected at $\infty$.

This paper further investigates how the hypotheses of the original theorem may be adjusted without affecting the conclusion that $G$ is simply connected at $\infty$. In particular, we shift the finitely presented condition on $H$ to a normal subgroup of $H$. Assume that $N \triangleleft H \triangleleft G$ are infinite groups with $G / H$ infinite and $N$ and $G$ finitely presented. Jackson and Houghton's theorem suggests that the additional hypothesis of $G / H$ being 1-ended might ensure the conclusion. (This question is raised by Mihalik in [10].) However, $\S \mathrm{V}$ will present finitely presented groups showing that no hypothesis on $G / H$ (that can be satisfied by a finitely presented group) is sufficient to ensure that $G$ is simply connected at $\infty$. (See Corollary V.1.1.)

With $N \triangleleft H \triangleleft G$ as above, Jackson and Houghton's theorem also suggests that the additional hypothesis of $N$ being 1-ended may be sufficient for $G$ to be simply connected at $\infty$. This yields the

Main Theorem. Let $N \triangleleft H \triangleleft G$ be groups with $N$ and $G$ finitely presented and $N$ 1-ended. If $G / H$ or $H / N$ is infinite, then $G$ is simply connected at $\infty$.

$\S I V$ will exhibit a class of finitely presented groups that are simply connected at $\infty$ by the Main Theorem. Further, it will be shown that the theorem of Jackson and Houghton does not apply to these groups.

The simple connectivity at $\infty$ of a finitely presented group is an important group invariant. In [4] Geoghegan and Mihalik show that if a finitely presented group $G$ is simply connected at $\infty$ then $H^{2}(G ; \mathscr{Z} G)=0$. The converse of this is false. Davis [3] presents examples of groups $G$ that are not simply connected at $\infty$ but satisfy $H^{2}(G ; \mathscr{Z} G)=0$. Thus, simple connectivity at $\infty$ is a stronger group invariant than second cohomology.

Simple connectivity at $\infty$ of finitely presented groups has applications in many settings. To mention a couple, Hillman [5] uses simple connectivity at $\infty$ in his results concerning 2 -knot groups. Also, simple connectivity at $\infty$ is used in [ 8 and 1] to show that universal covers of certain closed 3-manifolds are homeomorphic to Euclidean 3-space.

This paper has been written under the auspices of M. Mihalik whose assistance and guidance is hereby acknowledged and greatly appreciated. This paper will also serve as partial fulfillment of the requirements towards a Ph.D. at Vanderbilt University.

\section{Preliminaries}

The purpose of this section is to introduce terminology, constructions, and elementary facts for the results to follow.

II.1 Ends of groups. Let $X$ be a connected locally finite CW-complex. A continuous map $f:[0, \infty) \rightarrow X$ is proper if $C$ compact in $X$ implies that $f^{-1}(C)$ 
is compact in $[0, \infty)$. Let $A$ denote the set of all proper maps $[0, \infty) \rightarrow X$. Define an equivalence relation $\approx$ on $A$ by $r \approx s$ if for each compact $C$ in $X$ there exists an integer $N$ such that $r([N, \infty))$ and $s([N, \infty))$ are contained in the same unbounded path component of $X-C$. (A path component of $X-C$ is unbounded if it is contained in no compact subset of $X$.) The set of ends of $X$ is $A / \approx$. The number of ends of $X$ is the cardinality of $A / \approx$. Observe if $X$ is 1 -ended and $C$ is compact in $X$ then $X-C$ has exactly one unbounded path component.

Given a finitely generated group $G$, the number of ends of $G$ is the number of ends of the graph of $G$ with respect to a finite generating set. This definition is independent of which finite generating set is used.

For later use, if $G$ contains a finitely generated, 1-ended, subnormal subgroup, then $G$ is 1-ended. (See, for instance, Cohen [2].)

II.2 Constructions. There are two constructions which will be important later. The first, a 2-complex, is the universal cover of a 2-complex with a specified fundamental group. The proof of the theorem will take place in such a space. The second construction is a subcomplex of the first which will eventually allow for a convenient description of the necessary compact sets.

Let $G$ be a group with finite presentation $p=\left\langle g_{1}, \ldots, g_{\alpha} ; r_{1}, \ldots, r_{\beta}\right\rangle$. Let $*$ be a vertex. For each generator $g_{i}$, attach a loop to $*$. Now attach 2-cells to these loops according to the relations $\left\{r_{i}\right\}_{i=1}^{\beta}$. This space, $X_{p}$, is the standard 2-complex with fundamental group $G$ with respect to the presentation $p$.

Let $X$ denote the universal cover of $X_{p}$. The 1-skeleton of $X$ is the graph of $G$ with respect to the generating set $\left\{g_{1}, \ldots, g_{\alpha}\right\}$. Hence the edges of $X$ correspond to the group elements $g_{1}^{ \pm 1}, \ldots, g_{\alpha}^{ \pm 1}$ and the vertices of $X$ correspond to the elements of $G$. Any edge-path $e_{1} \cdots e_{k}$ of $X$ corresponds to some group element $e_{1}^{\prime} \cdots e_{k}^{\prime}$ where $e_{i}^{\prime} \in\left\{g_{1}^{ \pm 1}, \ldots, g_{\alpha}^{ \pm 1}\right\}$. However, determining an edge path of $X$ from some $e_{1}^{\prime} \cdots e_{k}^{\prime}$ requires the specification of an initial vertex since $e_{1}^{\prime} \cdots e_{k}^{\prime}$ at a vertex $v$ determines a different edge-path than $e_{1}^{\prime} \cdots e_{k}^{\prime}$ at another vertex $w$ (though these edge-paths differ by the covering transformation taking $v$ to $w$ ). When the presentation is understood, $X$ will be called the universe of $G$.

$X$ is obtained from the graph of $G$ by attaching 2-cells, at each vertex, according to the relations $r_{1}, \ldots, r_{\beta}$. Observe that the number of ends of $X$ is the same as the number of ends of $G$.

For the second construction, let $A$ be a subcomplex of $X$. Let $V(A)$ be the set of all vertices in $A$ together with all the vertices in $X$ that can be reached by a path of length 1 from a vertex in $A$. Define the Star of $A$, denoted $\mathrm{St}(A)$, to be the largest subcomplex of $X$ whose vertices lie in $V(A)$. Clearly $A$ lies in $\operatorname{St}(A)$. Define the $N$ th star of $A(N=1,2, \ldots)$ recursively as

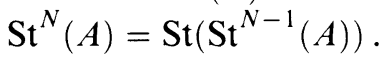


Observe that the star of a compact (i.e. finite) subcomplex in $X$ is again a compact subcomplex and hence when $A=\{v\}$ for some vertex $v \in X$, the sequence $\{v\} \subset \operatorname{St}(\{v\}) \subset \cdots \subset \mathrm{St}^{N}(\{v\}) \subset \cdots$ is an exhausting sequence of compact sets in $X$.

II.3 Simply connected at $\infty$. A topological space $X$ is simply connected at $\infty$ if for each compact set $C$ in $X$ there exists a compact set $D$ in $X$ such that if $\gamma$ is a loop in $X-D$ then $\gamma$ is homotopically trivial in $X-C$. This definition extends to finitely presented groups via the first construction in II. 2 . A finitely presented group $G$ is simply connected at $\infty$ if the universal cover of the standard 2-complex with fundamental group $G$ with respect to some finite presentation is simply connected at $\infty$. This definition is independent of the finite presentation chosen for $G$.

When considering the simple connectivity at $\infty$ of a finitely presented group, it suffices to consider only edge loops in the universal cover. This observation will be implicit in the proof in the next section, albeit not explicitly mentioned.

II.4 A preliminary fact. The preliminary section closes with an elementary fact: If $X$ is a connected, locally connected, locally compact Hausdorff space and $C$ is compact in $X$, then $C$ union the bounded path components of $X-C$ is compact in $X$. Recall from II. 1 that if $X$ is 1 -ended and $C$ is compact in $X$, then $X-C$ has exactly one unbounded path component. Later, the following will be used: If $X$ is 1 -ended and $C$ is compact in $X$, then $C$ union the bounded components of $X-C$ is compact and its complement is unbounded and path connected.

\section{THE THEOREM}

This section presents the main theorem. The proof begins by establishing the necessary notation and then follows from a short succession of claims. The idea of the proof is to show that a certain type of loop is trivial outside a compact set and that every loop is homotopic outside the compact set to a loop of the appropriate type.

Main Theorem. Let $N \triangleleft H \triangleleft G$ be groups with $N$ and $G$ finitely presented and $N$ 1-ended. If $G / H$ or $H / N$ is infinite then $G$ is simply connected at $\infty$.

Proof. First consider the case when $G / H$ is finite and $H / N$ is infinite. Then $H$ is finitely presented since $G$ is. Thus $G$ is simply connected at $\infty$ iff $H$ is. But since $N$ is finitely presented and 1-ended, $H$ is simply connected at $\infty$ by the theorem of Jackson and Houghton.

We may now assume that $G / H$ is infinite.

Let $\left\langle n_{1}, \ldots, n_{\alpha} ; r_{1}, \ldots, r_{\beta}\right\rangle$ be a finite presentation for $N$. This extends to a finite presentation $\left\langle n_{1}, \ldots, n_{\alpha}, g_{1}, \ldots, g_{\delta} ; r_{1}, \ldots, r_{\beta}, s_{1}, \ldots, s_{\varepsilon}\right\rangle$ of $G$ where $\left\{g_{1} H, \ldots, g_{\delta} H\right\}$ is a generating set for $G / H$. 
Let $X$ denote the universe of $G$. At each vertex $v$ in $X$, let $v N$ denote the universe of $N$ based at $v$. If $w$ is another vertex of $X$, then $v N=w N$ iff $v=n w$ in $G$ for some $n$ in $N$.

Let $\pi: X \rightarrow X / H$ be the projection map. Observe that if $v$ is a vertex of $X$ then every vertex of $v N$ projects to $\pi(v)$.

Let $C$ be compact in $X$. Without loss of generality, there exists a vertex $v_{0}$ in $X$ and integer $K$ such that $C$ is the union of $\operatorname{St}^{K}\left(\left\{v_{0}\right\}\right)$ and the bounded path components of $X-\mathrm{St}^{K}\left(\left\{v_{0}\right\}\right)$. Then for each vertex $v$ in $X, v N \cap C \neq \phi$ iff $C$ contains a vertex of $v N$.

Claim 1. There exists a compact set $D$ in $X$ such that if $\gamma$ is an edge loop in $v N-D$ for some vertex $v$ in $X$ then $\gamma$ is a trivial loop in $X-C$.

If $v N \cap C=\phi$ then $\gamma$ is trivial in $v N$ and hence in $X-C$.

Assume $v N \cap C \neq \phi$.

Fix a vertex $u_{0}$ in $X / H-\pi(C)$. At each vertex $u$ in $\pi(C)$, let $p_{u}$ be an edge path in $X / H$ from $u$ to $u_{0}$. Since $\pi(C)$ is compact, it contains only finitely many vertices. Let $L$ denote the maximum length of the $p_{u}$ 's.

If $x$ is a vertex of $X$ such that $\pi(x)$ lies in $\pi(C)$, let $p_{x}$ denote the lift of $p_{\pi(x)}$ to $x$. Notationally, let $\bar{x}$ denote the terminal vertex of $p_{x}$. Observe $\pi(\bar{x})=u_{0}$ misses $\pi(C)$ so edge loops in $\bar{x} N$ are trivial in $X-C$.

Let $F$ denote the free group on $\left\{n_{1}, \ldots, n_{\alpha}, g_{1}, \ldots, g_{\delta}\right\}$. Let $\mathscr{L}$ be the subset of $F$ consisting of all words of the form $p e_{1}^{-1} p^{-1} e_{2} p e_{1} p^{-1}$ where $p$ is a word in $F$ of length at most $L$ and $\left\{e_{1}, e_{2}\right\}$ is contained in $\left\{n_{1}, \ldots, n_{\alpha}, n_{1}^{-1}\right.$, $\left.\ldots, n_{\alpha}^{-1}\right\}$. Observe $\mathscr{L}$ is a finite set. Further if $g$ is in $\mathscr{L}$ then the image of $g$ in $G$ lies in $N$. Choose $w(g)$ in $F$ to be a word in $\left\{n_{1}, \ldots n_{\alpha}\right\}$ such that $g w(g)^{-1}$ lies in the kernel of the presentation for $G$. If $x$ is a vertex of $X$ then $g w(g)^{-1}$ determines an edge loop at $x$ of length at most $4 L+3+w$ where $w \equiv$ $\max _{g \in \mathscr{L}}$ (length of $w(g)$ ).

Let $M$ be an integer such that if $x$ is a vertex of $X$ then every edge loop at $x$ of length at most $4 L+3+w$ is trivial in $\operatorname{St}^{M}(\{x\})$.

If $x$ is a vertex of $X$, let $C_{x}$ denote $\mathrm{St}^{M}(C)$ intersected with $x N$ union the bounded path components of $x N-\mathrm{St}^{M}(C)$. Then $C_{x}$ is compact. Further, only finitely many such $C_{x}$ are nonempty since $\mathrm{St}^{M}(C)$ is compact. Let $\widetilde{C}$ denote the compact set consisting of the union of $\mathrm{St}^{M}(C)$ with the bounded components of $X-\mathrm{St}^{M}(C)$ and all the $C_{x}$ 's. Define $D$ to be $\operatorname{St}^{L}(\widetilde{C})$. Since $\widetilde{C}$ is compact so is $D$. Observe that if $x$ is a vertex of $X-D$ such that $\pi(x)$ lies in $\pi(C)$ then $\bar{x}$ (=terminus of $p_{x}$ ) lies in the unbounded path component of $\bar{x} N-\mathrm{St}^{M}(C)$.

Subclaim 1.1. Let $x$ be a vertex in $X-D$ such that $\pi(x)$ lies in $\pi(C)$. Let $r$ be an edge path in $x N-D$ from $x$ to a vertex $y$. Let $q_{1}$ be an edge path in $\bar{x} N-\mathrm{St}^{M}(C)$ from $\bar{x}$ to a vertex $\hat{x}$. If both occurrences of $r$ in 


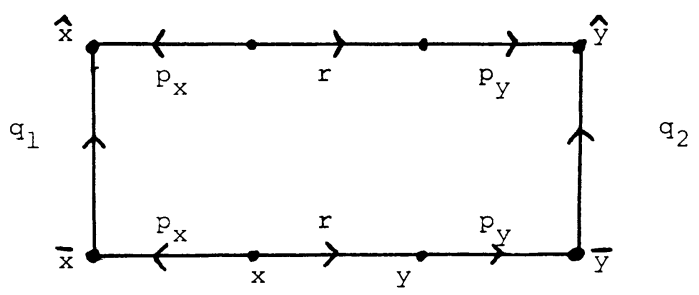

FIGURE 1

the edge path $p_{y}^{-1} r^{-1} p_{x} q_{1} p_{x}^{-1} r p_{y}$ (from $\bar{y}$ to a vertex $\hat{y}$ ) lie in $X-D$, then there exists an edge path $q_{2}$ in $\bar{y} N-\mathrm{St}^{M}(C)$ from $\bar{y}$ to $\hat{y}$. Moreover, for any such $q_{2}$ the edge path $r$ at $x$ is homotopic (rel endpoints) to the edge path $p_{x} q_{1} p_{x}^{-1} r p_{y} q_{2}^{-1} p_{y}^{-1}$. Further, the homotopy takes place in $X-C$. (See Figure 1.)

Observe that $p_{x}$ and $p_{y}$ are both lifts of $p_{\pi(x)}=p_{\pi(y)}$ and thus correspond to the same element of $G$. Hence $p_{y}^{-1} r^{-1} p_{x}$ corresponds to an element of $H$ $(H \triangleleft G)$ and $p_{y}^{-1} r^{-1} p_{x} q_{1} p_{x}^{-1} r p_{y}$ corresponds to an element of $N(N \triangleleft H)$. This yields that $\hat{y}$ is a vertex of $\bar{y} N$. By hypothesis, $\hat{y}$ and $\bar{y}$ both lie in the unbounded path component of $\bar{y} N-\mathrm{St}^{M}(C)$. Hence there exists an edge path $q_{2}$ in $\bar{y} N-\mathrm{St}^{M}(C)$ from $\bar{y}$ to $\hat{y}$.

It suffices to verify the case when $r$ consists of a single edge as Subclaim 1.1 then follows by a straightforward induction on the edge length of $r$.

Suppose $r$ corresponds to an element of $\left\{n_{1}, \ldots, n_{\alpha}, n_{1}^{-1}, \ldots, n_{\alpha}^{-1}\right\}$. We can write $q_{1}$ as an edge sequence $e_{1}, \ldots, e_{k}$ where each $e_{i}$ corresponds to an element of $\left\{n_{1}, \ldots, n_{\alpha}, n_{1}^{-1}, \ldots, n_{\alpha}^{-1}\right\}$. For each $i$, the element $f_{i} \equiv$ $p_{y}^{-1} r^{-1} p_{x} e_{i} p_{x} r p_{y}$ is in $\mathscr{L}$. Thus the edge path $p_{y}^{-1} r^{-1} p_{x} q_{1} p_{x}^{-1} r p_{y}$ is homotopic to $w\left(f_{1}\right) w\left(f_{2}\right) \cdots w\left(f_{k}\right)$ in $\mathrm{St}^{M}\left(q_{1}\right)$, hence missing $C$. (See Figure 2.)

So, the edge path $r$ at $x$ is homotopic in $X-C$ to $p_{x} q_{1} p_{x}^{-1} r p_{y} w\left(f_{k}\right)^{-1} \ldots$ $w\left(f_{1}\right)^{-1} p_{y}^{-1}$. Since $q_{2} w\left(f_{k}\right)^{-1} \cdots w\left(f_{1}\right)^{-1}$ is an edge loop in $\bar{y} N$ and $\bar{y} N \cap C=$ $\phi, q_{2}^{-1}$ is homotopic missing $C$ to $w\left(f_{k}\right)^{-1} \cdots w\left(f_{1}\right)^{-1}$ and Subclaim 1.1 follows.

Observe in Subclaim 1.1 that if $r$ is an edge loop then $x=y, \bar{x}=\bar{y}$, and $\hat{x}=\hat{y}$. Also, when $r$ is an edge loop $q_{2}$ can be chosen to be $q_{1}$.

Let $\gamma$ be an edge loop in $v N-D$ based at the vertex $x$. Let $q_{1}$ be an edge path in $\bar{x} N-\mathrm{St}^{M}(C)$ from $\bar{x}$ to a vertex $\hat{x}$ chosen so that the edge loop $p_{x}^{-1} \gamma p_{x}$ at $\hat{x}$ lies in $X-D$ and is a trivial loop in $X-C$.

By Subclaim 1.1 (taking $r=\gamma$ ), $\gamma$ is homotopic in $X-C$ to the edge loop at $x$ given by $p_{x} q_{1} p_{x}^{-1} \gamma p_{x} q_{1}^{-1} p_{x}^{-1}$. Since $p_{x}^{-1} \gamma p_{x}$ in this loop is trivial in $X-C$ we conclude that $p_{x} q_{1} p_{x}^{-1} \gamma p_{x} q_{1}^{-1} p_{x}^{-1}$ (and hence $\gamma$ itself) is a trivial loop in $X-C$. 


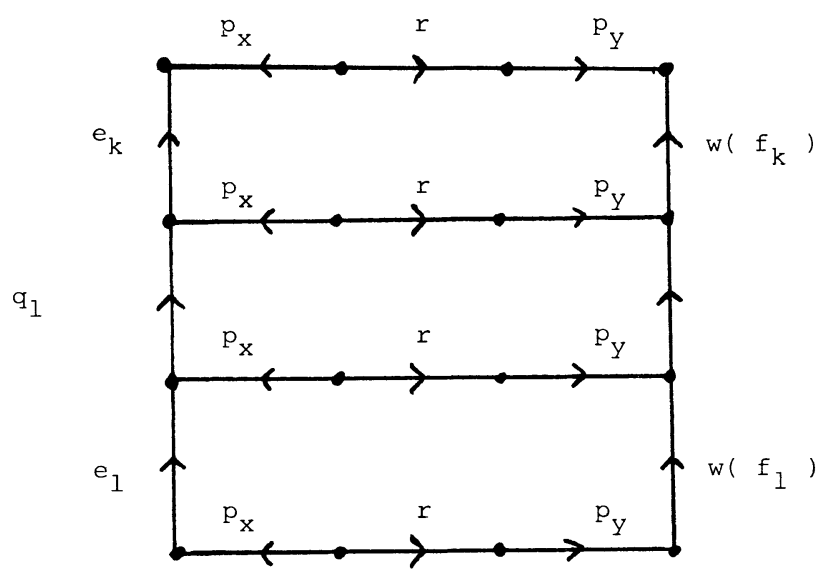

FIGURE 2

Claim 1 is now shown.

Let $D_{1}$ denote $\mathrm{St}^{M}(D)$ union the bounded path components of $x N-\mathrm{St}^{M}(D)$ for all vertices $x$ in $X$.

Claim 2. Let $p$ be an edge path in $X$ of length one (so $p$ corresponds to a generator or the inverse of a generator of $G)$. Let $r, q_{1}, q_{2}$ be edge paths with edges corresponding to elements of $\left\{n_{1}, \ldots, n_{\alpha}, n_{1}^{-1}, \ldots, n_{\alpha}^{-1}\right\}$. If $p r^{-1} p^{-1} q_{1} p r p^{-1} q_{2}$ is an edge loop in $X-D_{1}$, with both occurrences of $r$ lying in $X-\operatorname{St}\left(D_{1}\right)$, then the loop is trivial in $X-C$.

The proof of Claim 2 is omitted as it follows from Claim 1 and an induction argument on the edge length of $r$ that parallels the proof of Subclaim 1.1. The hypotheses on $r$ allow for the construction of a homotopy between the original loop and a loop in $X-D$ that is seen to be trivial by Claim 1 .

Let $D_{2}$ denote $\operatorname{St}\left(D_{1}\right)$ union the bounded path components of $x N-\operatorname{St}\left(D_{1}\right)$ for all vertices $x$ in $X$. Observe that if $x$ is a vertex of $X$ then $x N-D_{2}$ consists of the unbounded path component of $x N-\operatorname{St}\left(D_{1}\right)$. Thus, any two vertices in $x \mathrm{~N}-\mathrm{D}_{2}$ can be joined by an edge-path in $x \mathrm{~N}-\mathrm{D}_{2}$.

Claim 3. Let $p$ be an edge path in $X$. Let $r, q_{1}, q_{2}$ be edge paths with edges corresponding to elements of

$$
\left\{n_{1}, \ldots, n_{\alpha}, n_{1}^{-1}, \ldots, n_{\alpha}^{-1}\right\}
$$

If $p r^{-1} p^{-1} q_{1} \cdot p r p^{-1} q_{2}$ is an edge loop in $X-D_{2}$ with both occurrences of $r$ lying in $X-\operatorname{St}\left(D_{2}\right)$, then the loop is trivial in $X-C$.

The proof proceeds by induction on the edge length of $p$.

If $p$ has length zero then $r^{-1} q_{1} r q_{2}$ is an edge loop in $x N-D$ for some vertex $x$ in $X$. By Claim 1 this is trivial in $X-C$.

If $p$ has length one then Claim 3 follows by Claim 2 .

Assume $p$ has length at least two. Let $e$ denote the first edge of $p$ and let $p-e$ denote the rest of $p$. To avoid notational confusion define $p_{1}=p$ and 
$p_{2}=p$ so the loop can be written

$$
e\left(p_{1}-e\right) r^{-1}\left(p_{1}-e\right)^{-1} e^{-1} q_{1} e\left(p_{2}-e\right) r\left(p_{2}-e\right)^{-1} e^{-1} q_{2} .
$$

At the vertex connecting $e$ with $p_{1}-e$, let $\rho_{1}$ be an edge path with edges corresponding to elements of $\left\{n_{1}, \ldots, n_{\alpha}, n_{1}^{-1}, \ldots, n_{\alpha}^{-1}\right\}$ that misses $D_{2}$ and terminates at a vertex chosen such that the edge loop

$$
\left(p_{1}-e\right) r^{-1}\left(p_{1}-e\right)^{-1} e^{-1} q_{1} e\left(p_{2}-e\right) r\left(p_{2}-e\right)^{-1} e^{-1} q_{2} e
$$

at this vertex lies in $X-\operatorname{St}\left(D_{2}\right)$ and is trivial in $X-C$ (see Figure 3).

The vertex in the original loop connecting $\left(p_{1}-e\right)^{-1}$ and $e^{-1}$ and the corresponding vertex in the loop at the end of $\rho_{1}$ both lie in the unbounded path component of $v N-D_{2}$ for some vertex $v$ in $X$. Let $\rho_{2}$ be an edge path in $v N-D_{2}$ connecting these vertices (see Figure 3).

Similarly, there exists an edge path $\rho_{3}$ in some $v N-D_{2}$ from the vertex connecting $e$ with $p_{2}-e$ in the original loop and the corresponding vertex in the loop at the end of $\rho_{1}$. Likewise, there exists an edge path $\rho_{4}$ in some $v N-D_{2}$ from the vertex connecting $\left(p_{2}-e\right)^{-1}$ and $e^{-1}$ in the original loop and the corresponding vertex in the loop at the end of $\rho_{1}$ (see Figure 3 ).

The edge loops $e^{-1} q_{1}^{-1} e \rho_{2}^{-1} e^{-1} q_{1} e \rho_{3}$ and $e^{-1} q_{2} e \rho_{1}^{-1} e^{-1} q_{2}^{-1} e \rho_{4}$ are trivial in $X-C$ by Claim 2 (e.g., for the former loop take in Claim 2: $p=e^{-1}$, $r=q_{1}, q_{1}=\rho_{2}^{-1}$, and $\left.q_{2}=\rho_{3}\right)$.

The edge loop $\left(p_{1}-e\right) r^{-1}\left(p_{1}-e\right)^{-1} \rho_{2}\left(p_{1}-e\right) r\left(p_{1}-e\right)^{-1} \rho_{1}^{-1}$ is trivial in $X-C$ by the induction hypothesis.

Thus the original loop is homotopic in $X-C$ to the edge loop

$$
\left(p_{2}-e\right) r^{-1}\left(p_{2}-e\right)^{-1} \rho_{3}\left(p_{2}-e\right) r\left(p_{2}-e\right)^{-1} \rho_{4}^{-1} \text {. }
$$

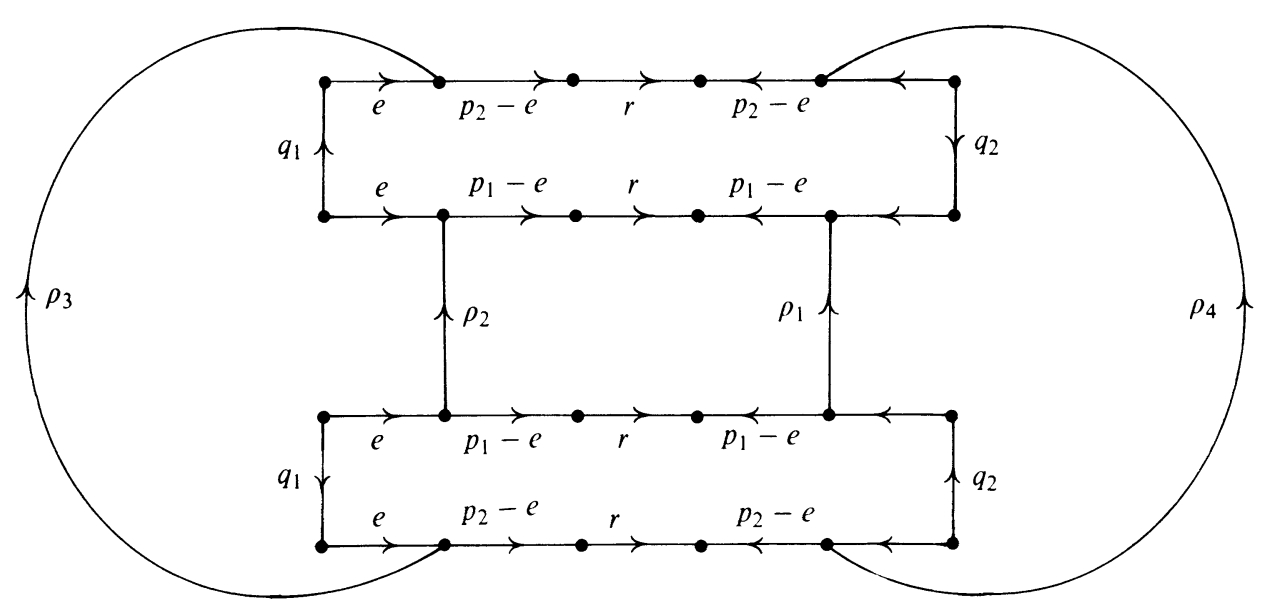

FIGURE 3 


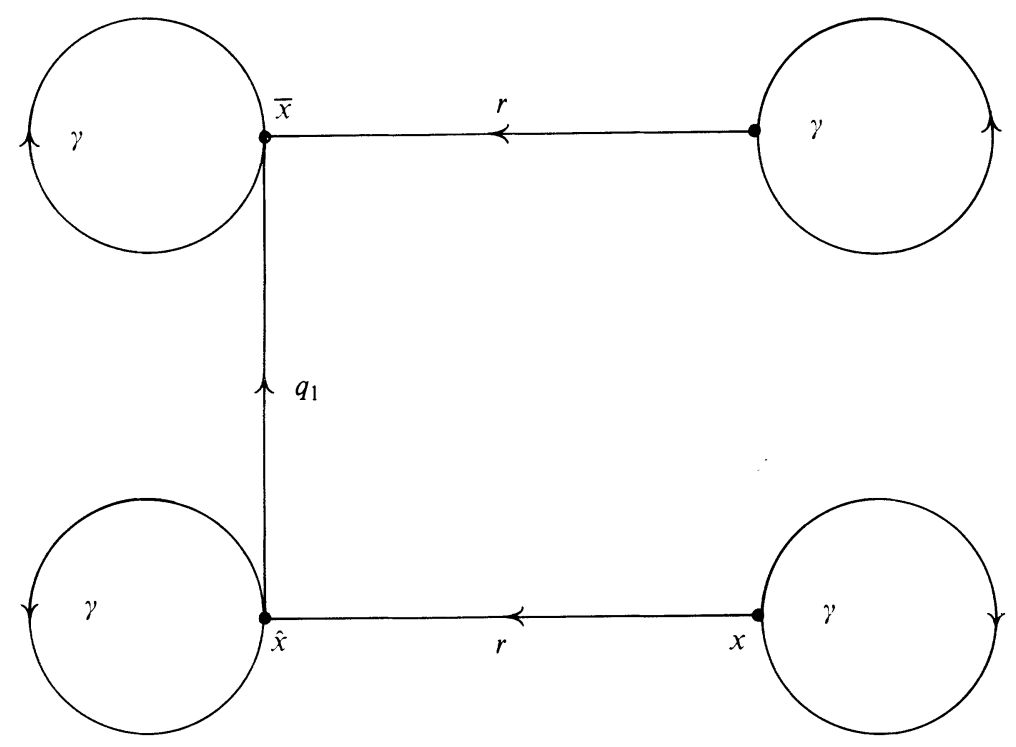

FIGURE 4

Claim 3 now follows by realising that the edge loop

$$
\left(p_{2}-e\right) r^{-1}\left(p_{2}-e\right)^{-1} \rho_{3}\left(p_{2}-e\right) r\left(p_{2}-e\right)^{-1} \rho_{4}^{-1}
$$

is trivial in $X-C$, again using the induction hypothesis.

To complete the Theorem, let $D_{3}$ denote $\operatorname{St}\left(D_{2}\right)$ union the bounded path components of $x N-\operatorname{St}\left(D_{2}\right)$ for all vertices $x$ in $X$.

Let $\gamma$ be an edge loop in $X-D_{3}$ based at the vertex $x$. Let $r$ be an edge path in $x N-\operatorname{St}\left(D_{2}\right)$ from $x$ to a vertex $\hat{x}$ where $\hat{x}$ is chosen so that $\gamma$ at $\hat{x}$ misses $D_{3}$ and is trivial in $X-C$.

From $\hat{x}$, let $q$ be an edge path in $x N-\operatorname{St}\left(D_{2}\right)$ to a vertex $\bar{x}$ chosen so that $\gamma r^{-1} \gamma^{-1}$ at $\bar{x}$ lies in $X-D_{3}$ and both occurrences of $\gamma$ are trivial in $X-C$ (see Figure 4).

Clearly $\gamma$ at $x$ is homotopic in $X-C$ to the edge loop at $x$ given by $\gamma r \gamma^{-1} q \gamma r^{-1} \gamma^{-1}\left(r q^{-1} r^{-1}\right)$. But the latter is an edge loop in $X-\operatorname{St}\left(D_{2}\right)$ and thus trivial in $X-C$ by Claim 3. Hence $\gamma$ is also trivial in $X-C$ and the proposition follows.

As a corollary to the Main Theorem we generalize a special case of Mihalik's theorem.

Corollary. Let $N \triangleleft H \triangleleft G$ be infinite groups with $N$ and $G$ finitely presented, $H$ abelian, $G / H$ infinite, and $H / N$ not locally finite. Then $G$ is simply connected at $\infty$.

Since $N$ is infinite, abelian, and finitely generated, $N$ contains a copy of $\mathscr{Z}$. Since $H / N$ is abelian and not locally finite, $H / N$ also contains a copy of 
$\mathscr{Z}$ distinct from the $\mathscr{Z}$ in $N$. Thus $H$ contains a copy of $\mathscr{Z} \oplus \mathscr{Z}$ which is normal in $H$ since $H$ is abelian.

\section{AN EXAMPLE}

The purpose of this section is to exhibit a class of groups applicable to the Main Theorem but not to Jackson and Houghton's theorem.

Notationally, for an integer $p>1$ let $\mathscr{Z}\left[\frac{1}{p}\right]$ denote the group of rationals with denominators a power of $p$.

Let $G=\left\langle x, y, t ;[x, y], t^{-1} x t=x^{p}, t^{-1} y t=y^{q}\right\rangle$ where $p$ and $q$ are integers greater than 1 . Let $N$ denote the subgroup of $G$ generated by $x$ and $y$, and let $H$ be the normal subgroup of $G$ generated by $x$ and $y$.

A straightforward application of the Reidemeister-Schreier method (see for instance [9]) verifies that $H \cong \mathscr{Z}\left[\frac{1}{p}\right] \oplus \mathscr{Z}\left[\frac{1}{p}\right]$ where $N$ corresponds to the subgroup generated by $0 \oplus 1$ and $1 \oplus 0$, and in $G$ we have $t \frac{1}{p^{i}} t^{-1}=\frac{1}{p^{i-1}}$ and $t \frac{1}{q^{i}} t^{-1}=\frac{1}{q^{i-1}}$.

We can now observe that $N \triangleleft H \triangleleft G$ with $N(\cong \mathscr{Z} \oplus \mathscr{Z})$ finitely presented and 1 -ended, $G$ finitely presented and $G / H \cong \mathscr{Z}$ is infinite. Thus by the Main Theorem $G$ is simply connected at $\infty$.

Further observe that no finitely generated subgroup of $H$ can be normal in $G$. The following claim then assures that Jackson's and Houghton's theorem does not apply.

Claim. If $K \triangleleft G$ and $K$ does not lie in $H$, then $G / K$ is finite.

To see this, observe that every element of $G$ can be written in the form $h t^{m}$ for some $h$ in $H$ and integer $m$. If $K$ does not lie in $H$, there exists $h$ in $H$ and nonzero integer $m$ such that $h t^{m}$ lies in $K$. Thus $t^{m} K=h^{-1} K$ in the image of $H$ in $G / K$. Since $H$ is abelian and $t^{-|m|} x t^{|m|}$ is a power of $x$, the image of $x$ in $G / K$ has finite order. Likewise the image of $y$ in $G / K$ has finite order. Thus the image of $H$ in $G / K$ is an abelian torsion group. Hence the subgroup $\widetilde{H}$ of $G / K$ generated by the images of $h, x$, and $y$ is finite. The claim now follows by realizing that every element of $G / K$ can be written in the form $t^{\alpha} g t^{\beta} K$ where $g \in \widetilde{H}$ and $|\alpha|<m$ and $|\beta|<m$.

\section{COUNTEREXAMPLES}

The purpose of this section is to present the examples alluded to in the introduction. The first example will show (take $G_{1}=\mathscr{Z} \times \mathscr{Z}$ in Corollary V.1.1 for instance) that if $N \triangleleft H \triangleleft G$ are infinite groups with $N$ and $G$ finitely presented and $G / H$ is 1 -ended, then $G$ need not be simply connected at $\infty$. In fact, no condition on $G / H$ that can be achieved by a finitely presented group is sufficient for $G$ to be simply connected at $\infty$.

The second example will show that if $G$ is finitely presented, $H$ is finitely generated and 1-ended, and $H \triangleleft G$ with $G / H$ infinite, then $G$ need not be simply connected at $\infty$. The examples will be corollaries to the following result. 
Theorem V.1. Let $G_{1}$ and $G_{2}$ be finitely presented groups. If $F_{1}=\mathscr{Z} * G_{1}$, and $F_{2}=\mathscr{Z} * G_{2}$, then $F_{1} \times F_{2}$ is not simply connected at $\infty$.

Proof. For $i=1$ or 2 , choose a finite presentation for $G_{i}$. This enlarges to a finite presentation of $F_{i}$ by adding a new generator $a_{i}$.

Let $X_{i}$ denote the universe of $F_{i}$. Within $X_{i}$, let $Z_{i}$ denote the graph of the subgroup $\left\langle a_{i}\right\rangle$ of $F_{i}$ generated by $a_{i}$ and based at the identity vertex of $X_{i}$.

Using the induced presentation for $F_{1} \times F_{2}$, let $X$ denote the universe of $F_{1} \times F_{2}$. (Observe that $X$ is the 2-skeleton of $X_{1} \times X_{2}$.) We may identify $Z_{1} \times Z_{2}$ with the graph (and appropriate 2-cells) of the subgroup of $F_{1} \times F_{2}$ generated by $a_{1}$ and $a_{2}$ and based at the identity vertex of $X$.

Let $C$ be the compact set in $Z_{1} \times Z_{2}$ consisting of a single point in the interior of the 2-cell at the identify vertex determined by the relation $a_{1}^{-1} a_{2}^{-1} a_{1} a_{2}$.

By construction, the canonical homomorphisms $F_{1} \rightarrow \mathscr{Z}$ and $F_{2} \rightarrow \mathscr{Z}$ induce a retraction of $X$ onto $Z_{1} \times Z_{2}$ such that $X-\left(Z_{1} \times Z_{2}\right)$ maps onto the 1 -skeleton of $Z_{1} \times Z_{2}$. In particular, $X-\left(Z_{1} \times Z_{2}\right)$ maps into $\left(Z_{1} \times Z_{2}\right)-C$.

Thus, if $D$ is compact in $X$ then an edge loop in $\left(Z_{1} \times Z_{2}\right)-D$ is trivial in $X-C$ iff it is trivial in $\left(Z_{1} \times Z_{2}\right)-C$. Since for any compact set $D$ in $X$ there exists an edge loop in $\left(Z_{1} \times Z_{2}\right)-D$ that is not trivial in $\left(Z_{1} \times Z_{2}\right)-C$, it follows that $X$ is not simply connected at $\infty$ and the theorem is shown.

Corollary V.1.1. Let $G_{1}$ be a finitely presented group. Then there exist groups $N \triangleleft H \triangleleft G$ with $N$ and $G$ finitely presented and $G / H \cong G_{1}$ such that $G$ is not simply connected at $\infty$.

Proof. Let $\left\langle g_{1}, \ldots, g_{\alpha} ; r_{1}, \ldots, r_{\beta}\right\rangle$ be a finite presentation for $G_{1}$. Choose $G=\left\langle x, g_{1}, \ldots, g_{\alpha}, a ; r_{1}, \ldots, r_{\beta},[x, a],\left[g_{1}, a\right], \ldots,\left[g_{\alpha}, a\right]\right\rangle$. Let $H$ be the normal subgroup of $G$ generated by $x$ and $a$ so that $G / H \cong G_{1}$. Let $N$ be the subgroup of $H$ generated by $a$. Then $N \triangleleft H$ (since $N \triangleleft G$ ) and $N \cong \mathscr{Z}$.

The corollary now follows by observing that $G \cong\left(\mathscr{Z} * G_{1}\right) \times \mathscr{Z}$ which is not simply connected at $\infty$ by Theorem V.1.

Corollary V.1.2. For each infinite finitely presented group $G_{1}$, there exists a 1ended finitely generated group $H$ containing $G_{1}$ and a finitely presented group $G$ such that $H \triangleleft G$ of infinite index and $G$ is not simply connected at $\infty$.

Proof. Let $\left\langle g_{1}, \ldots, g_{\alpha} ; r_{1}, \ldots, r_{\beta}\right\rangle$ be a finite presentation for $G_{1}$. Let $H=$ $\left\langle y, z, g_{1}, \ldots, g_{\alpha} ; r_{1}, \ldots, r_{\beta},\left[g_{J}, y^{i} z y^{-i}\right] \quad(1 \leq J \leq \alpha, i\right.$ in $\left.\mathscr{Z})\right\rangle$. Observe that $H$ contains $G_{1}$.

Let $N$ be the subgroup of $H$ generated by $\left\{y^{i} z y^{-i}\right\}_{i=1}^{\infty}$. Then $N \triangleleft H$ and $N$ lies in the subgroup of $H$ generated by $y$ and $z$. This latter group has infinite index in $H$ so $H$ is 1-ended by Proposition 2.5 of [2].

Define an isomorphism $\phi: H \rightarrow H$ by $\phi(y)=y, \phi(z)=y z y^{-1}$, and $\phi\left(g_{J}\right)=g_{J}$ for $1 \leq J \leq \alpha$. 
Let $G=\left\langle y, z, g_{1}, \ldots, g_{\alpha} ; r_{1}, \ldots, r_{\beta},\left[g_{J}, y^{i} z y^{-i}\right], t^{-i} h t=\phi(h)\right\rangle$ where $1 \leq J \leq \alpha, i$ is in $\mathscr{Z}$, and $h$ is in $H$. (Observe that $H \triangleleft G$ of infinite index as claimed.) This reduces to the finite presentation $G=\left\langle y, z, g_{1}, \ldots, g_{\alpha}, t\right.$; $\left.r_{1}, \ldots, r_{\beta},\left[g_{J}, z\right],\left[g_{J}, t\right],[y, t], t^{-1} z t=y z y^{-1}\right\rangle$. The Tietze move adding the generator $a=t y$ (so $y=t^{-1} a$ ) yields that $G \cong\left(\mathscr{Z} * G_{1}\right) \times(\mathscr{Z} * \mathscr{Z})$ which completes the corollary.

\section{BIBLIOGRAPHY}

1. M. Brin and T. Thichstun, Open irreducible 3-manifolds which are end 1-movable, Topology 26 (1987), 211-233.

2. D. E. Cohen, Groups of cohomological dimension one, Lecture Notes in Math., vol. 245, Springer-Verlag, Berlin and New York, 1972.

3. M. Davis, Groups generated by reflections and aspherical manifolds not covered by Euclidean spaces, Ann. of Math. (2) 117 (1983), 293-324.

4. R. Geoghegan and M. L. Mihalik, A note on the vanishing of $H^{n}(G, \mathscr{Z} G)$, J. Pure Appl. Algebra 39 (1986), 301-304.

5. J. A. Hillman, Abelian normal subgroups of two-knot groups, Comment. Math. Helv. 61 (1986), 122-148.

6. C. H. Houghton, Cohomology and the behaviour at infinity of finitely presented groups, J. London Math. Soc. 15 (1977), 465-471.

7. B. Jackson, End invariants of group extensions, Topology 21 (1982), 71-81.

8. F. E. A. Johnson, Manifolds of homotopy type $K(\pi, 1)$. II, Proc. Cambridge Philos. Soc. 75 (1974), 165-173.

9. W. Magnus, A. Karrass, and D. Solitar, Combinatorial group theory, Interscience, 1966.

10. M. L. Mihalik, Solvable groups that are simply connected at infinity, Math. Z. 195 (1987), 79-87.

Department of Mathematics, Vanderbilt University, Nashville, Tennessee 37325

Current address: Center for Naval Analyses, 4401 Ford Avenue, P. O. Box 16268, Alexandria, Virginia 22302-0268 